\title{
Associação entre Percepção de Doença e Ansiedade, Depressão e Autoeficácia em Pessoas com HIV/Aids
}

\author{
Graziela Sousa Nogueira ${ }^{1}$ \\ Universidade de Brasilia, Brasília, DF, Brasil \\ Secretaria de Estado de Saúde do Distrito Federal, Brasília, DF, Brasil \\ Eliane Maria Fleury Seidl \\ Instituto de Psicologia da Universidade de Brasília, Brasília, DF, Brasil
}

\begin{abstract}
Resumo
A percepção de doença de pessoas que vivem com HIV/aids (PVHA) é uma variável importante, ainda pouco estudada em âmbito mundial. Objetivou-se investigar a associação entre percepção de doença e ansiedade, depressão e autoeficácia em pessoas que vivem com HIV/aids. A amostra foi constituída de 28 PVHA, que responderam aos questionários sociodemográfico e médico-clínico, Questionário de Percepção de Doenças Versão Breve (Brief IPQ), Escala Hospitalar de Ansiedade e Depressão (HADS) e Escala de Autoeficácia para Seguir Prescrição Antirretroviral. Procedeu-se a análises estatísticas dos dados, sendo realizados testes de correlação de Spearman. Observou-se correlação positiva estatisticamente significativa entre percepção de doença e ansiedade $(r=0,64 ; p<0,01)$ e depressão $(r=0,68$; $p<0,01)$. Também foi identificada correlação negativa estatisticamente significativa entre percepção de doença e autoeficácia $(r \mathrm{~s}=-0,41 ; p<0,05)$, sugerindo que quanto menor a autoeficácia percebida para aderir aos medicamentos antirretrovirais, maior a percepção de ameaça imposta pela doença. Conclui-se que há associação entre percepção de doença e variáveis psicológicas (ansiedade, depressão e autoeficácia) em PVHA.
\end{abstract}

Palavras-chave: Percepção de doença, variáveis psicológicas, HIV/aids.

\section{Association between Illness Perception and Anxiety, Depression and Self-Efficacy in People with HIVIAIDS}

\begin{abstract}
The illness perception of people living with HIV/AIDS (PLWHA) is an important variable, poorly studied worldwide. The aimed was investigate the association between illness perception and anxiety, depression and self-efficacy in people living with HIV/AIDS. The sample consisted of 28 PLWHA, that responded to sociodemographic and medical-clinical questionnaires, Brief Illness Perception Questionnaire (Brief IPQ), Hospital Anxiety and Depression Scale (HADS) and Self-efficacy for Following Anti-retroviral Prescription Scale. The authors conducted statistical analyzes of the data, been carried Spearman correlation tests. Was observed a statistically significant positive correlation between illness perception and anxiety $(r=.64 ; p<.01)$ and depression $(r=.68 ; p<.01)$. Has also identified a statistically significant negative correlation between self-efficacy and illness perception $(r=-.41 ; p<.05)$, suggesting that the higher self-efficacy perceived for adhere to antiretroviral drugs, larger perception of
\end{abstract}

Endereço para correspondência: Quadra 205, Praça Jandaia, Ed. Ingrid, Apto. 704, Águas Claras Sul, DF, Brasil 71925-000. Fone: (61) 3522-1300/(61) 8100-3537. E-mail: grazysousa@yahoo.com.br Apoio financeiro: Conselho Nacional de Desenvolvimento Científico e Tecnológico (CNPq). 
threat posed by the disease. It was concluded that there is an association between illness perception and psychological variables (anxiety, depression and self-efficacy) in PLWHA.

Keywords: Illness perception, psychological variables, HIV/AIDS.

\section{Asociación entre la Percepción de la Enfermedad y la Ansiedad, la Depresión y la Auto-Eficacia en las Personas con VIH/SIDA}

\section{Resumen}

La percepción de la enfermedad de las personas que viven con el VIH/SIDA (PVVS) es una variable, ha sido poco estudiada en todo el mundo. El objetivo fue investigar la asociación entre la percepción de la enfermedad y la ansiedad, la depresión y la auto-eficacia en personas que viven con el VIH/SIDA. La muestra consistió en 28 PVVS, que respondieron a cuestionarios sociodemográficos y médicos-clínicos, Cuestionario de Perception de la Enfermidad Versión Breve, Escala Hospitalaria de Ansiedad y Depresión y la Escala de Autoeficacia para Tomar los Antirretrovirales. Los autores realizaron análisis estadísticos de los datos y conducidas pruebas de correlación de Spearman. Se observó una correlación positiva estadísticamente significativa entre percepción de la enfermedad y ansiedad $(r=.64 ; p<.01) \mathrm{y}$ depresión $(r=.68 ; p<.01)$. También se ha identificado una correlación negativa estadísticamente significativa entre la autoeficacia y la percepción de la enfermedad $(r=-.41 ; p<.05)$, lo que sugiere que quanto mayor la autoeficacia percibida para adherirse a los medicamentos antirretrovirales, mayor la percepción de amenaza que representa la enfermedad. De ello se desprende que existe una asociación entre la percepción de la enfermedad y variables psicológicas (ansiedad, depresión y auto-eficacia) en las PVVS.

Palabras clave: Percepción de la enfermedad, variables psicológicas, VIH/SIDA.

A aids é atualmente uma pandemia, sendo que aproximadamente 35 milhões de pessoas estão infectadas no plano mundial. Trata-se de um fenômeno global, com prevalência variável em diferentes regiões do mundo (Joint United $\mathrm{Na}$ tions Programme on HIV/AIDS, 2014).

No Brasil, informações do Departamento Nacional de DST, Aids e Hepatites Virais revelam que cerca de 734 mil casos de aids foram notificados, desde o início da epidemia até dezembro de 2013, sendo que foram registrados 278.306 óbitos. Somente no Distrito Federal, desde o início da epidemia até junho de 2014, foram notificados 10.107 casos de aids e 3.115 óbitos entre 1980 e 2013 (Ministério da Saúde, 2014). Ressalta-se, ainda, que a epidemia de aids no Brasil sofreu transformações no seu perfil epidemiológico, sendo hoje marcada pela heterossexualização, feminização e pauperização (Silva et al., 2010).

Desde 1996, a terapia antirretroviral (TARV) é acessível de forma universal no país, o que propiciou grandes avanços no tratamento da aids, pois ela retarda o seu desenvolvimento por meio da supressão viral e, em consequência, permite a restauração do sistema imunológico, reduzindo a morbimortalidade e melhorando a qualidade de vida de pessoas que vivem com HIV/aids - PVHA (Ministério da Saúde, 2008, 2012). No entanto, para que se alcance a eficácia terapêutica é necessário que haja adesão ao tratamento, expressa pelo uso igual ou superior a 95\% das doses prescritas (Alencar, Nemes, \& Velloso, 2008), o que constitui um desafio importante para pacientes, profissionais de saúde e gestores das políticas de HIV/aids no país.

Entre os fatores que podem facilitar a conduta de adesão, cita-se: conhecimento e compreensão sobre a enfermidade e o tratamento; vínculo com a equipe multiprofissional; presença de apoio social; e autoeficácia elevada para tomar os medicamentos antirretrovirais (Ministério da Saúde, 2008; Seidl, Melchíades, Farias, \& Brito, 2007). Já os fatores que podem dificultar a adesão incluem: complexidade do regime terapêutico e/ou ocorrência de efeitos colaterais; precarie- 
dade de suporte social, ocorrência de transtornos mentais como depressão e ansiedade; e relação insatisfatória com médico e demais profissionais de saúde (Ministério da Saúde, 2008; Seidl et al., 2007; Sharma, Khadga, Dhungana, \& Chitrakar, 2013). A relevância de variáveis psicológicas no processo de adesão à TARV remete à importância de serviços de saúde com equipes multidisciplinares especializadas na assistência a essa população, com destaque para a atuação do psicólogo da saúde.

\section{A Psicologia da Saúde no Contexto de Assistência a PVHA}

Segundo Straub (2014), a Psicologia da Saúde pode ser definida como "a aplicação de princípios e pesquisas psicológicas para a melhoria da saúde, a prevenção e o tratamento de doenças" (p. 3). Trata-se de uma área de contribuições profissionais, científicas e educacionais da psicologia para a promoção e manutenção da saúde (Matarazzo, 1980 conforme citado por Calvetti, Muller, \& Nunes, 2007). Assim, a psicologia da saúde estuda os fatores que determinam como as pessoas permanecem saudáveis, porque ficam doentes e como respondem à enfermidade e aos cuidados de saúde (Seidl \& Faustino, 2014).

Desta forma, a atuação do psicólogo no contexto de assistência à PVHA apresenta uma série de demandas. Apesar dos avanços conquistados com relação ao tratamento das PVHA, trata-se de um vírus de difícil controle, sem perspectivas de cura em curto e médio prazo. Tal panorama, aliado ao preconceito e discriminação social, sexual e afetiva, frequentemente vivenciados por pessoas soropositivas, contribuem para o sofrimento psicológico e a manifestação de transtornos mentais, como ansiedade e depressão, que podem contribuir para a não adesão ao tratamento. Um estudo brasileiro evidenciou tal associação, revelando que pessoas que apresentaram adesão insatisfatória à TARV, tinham mais sintomas de ansiedade e depressão em comparação com aquelas que tinham uma adesão adequada (Galvão, Gir, Fiuza, Cunha, \& Reis, 2013). Tucker, Burnam, Sherbourne, Kung e Gifford (2003) afirmam que pessoas com ansiedade e depressão tem duas vezes mais chances de terem pior ade- são em comparação com aquelas que não apresentam esses transtornos. Assim, a saúde mental das PVHA deve ser alvo constante de atenção e de intervenção da equipe de saúde, em especial de psicólogos, de modo a contribuir para o alívio do sofrimento psíquico e aumentar a probabilidade de adesão satisfatória à TARV (Ministério da Saúde, 2012; Seidl \& Faustino, 2014).

Ressalta-se que no âmbito da Psicologia da Saúde, pesquisadores têm proposto uma ampla gama de modelos teóricos, muitos deles aplicáveis ao contexto de pesquisa e prática associada ao HIV/aids. Esses modelos têm sido delineados com o intuito de compreender melhor a relação entre saúde e comportamento, objetivando a promoção da saúde, tratamento e gerenciamento da doença. Entre esses modelos destaca-se o modelo de autorregulação de Leventhal (Broadbent, Petrie, Main, \& Weinman, 2006; Cameron \& Leventhal, 2003).

\section{Modelo de Autorregulação de Leventhal}

O Modelo de Autorregulação de Leventhal é um modelo de senso comum, também conhecido como modelo cognitivo da doença, percepção de doença ou modelo de representação da doença (Singh, 2011). Ele é centrado nas crenças individuais sobre saúde/doença e nas respostas às ameaças de doença, partindo da premissa que o paciente é um agente ativo na resolução de seus problemas, respondendo de forma dinâmica à enfermidade, segundo sua interpretação e avaliação (Broadbent at al., 2006; Cameron \& Leventhal, 2003; Figueiras, 2008).

Segundo o modelo de autorregulação de Leventhal, as respostas às doenças seguem habitualmente três etapas (Pacheco-Huergo et al., 2012). A primeira é a representação cognitiva e emocional da ameaça à saúde ou interpretação: a pessoa doente, ao ser confrontada com uma enfermidade potencial por meio de pistas internas (ex. sintomas) ou externas (ex. informação), tenta atribuir um significado ao problema, com base nas suas cognições preexistentes sobre a enfermidade. Essas cognições organizam-se em cinco dimensões: (a) identidade - referese às ideias que o paciente possui sobre a sua doença, bem como à interpretação que faz dos 
sintomas; (b) causa - indica as atribuições que o paciente faz acerca das prováveis causas da sua doença, que podem focalizar fatores internos e externos; (c) dimensão temporal - refere-se à percepção de duração do problema de saúde, podendo ser aguda, crônica ou cíclica/episódica; (d) consequências - indicam as crenças que o indivíduo possui acerca da gravidade da doença e o provável impacto em aspectos sociais, físicos, psicológicos e financeiros; (e) cura/controle se refere ao quanto os pacientes acreditam que sua doença pode ser curada ou controlada, bem como reflete as crenças que o indivíduo possui sobre o tratamento da doença (Broadbent et al., 2006; Cameron \& Leventhal, 2003; C. M. Leite, 2011). Estudos com diferentes enfermidades crônicas demonstraram associação entre essas dimensões de representação da doença e aspectos psicológicos (Broadbent et al., 2006; Brooke, 2013), tais como modalidades de enfrentamento (Colleto \& Câmara, 2009; Sousa, Landeiro, Pires, \& Santos, 2011), humor (Arran, Craufurd, \& Simpson, 2014; Keeling, Bambrough, \& Simpson, 2013) e comportamento de adesão às recomendações médicas (Chen, Tsai, \& Lee, 2009; Kemppainen, Kim-Godwin, Reynolds, \& Spencer, 2008).

A segunda etapa do processo de autorregulação refere-se ao desenvolvimento e implementação das estratégias de enfrentamento. Nesta fase, a pessoa identifica e seleciona estratégias de enfrentamento que lhe permitem adquirir o equilíbrio físico e emocional ameaçado ou perdido. Por fim, na terceira fase ocorre a ponderação ou avaliação das estratégias de enfrentamento empregadas, com fins de adaptação à doença ou às suas consequências, visando a manutenção da qualidade de vida (Pacheco-Huergo et al., 2012).

Assim, a percepção de doença de PVHA é uma variável importante a ser considerada no contexto da prática profissional e da pesquisa sobre essa enfermidade. Observa-se que a população soropositiva para o HIV apresenta frequentemente crenças disfuncionais sobre a enfermidade. Tais interpretações distorcidas como considerar que a aids é sinônimo de morte, que não pode ser tratada e controlada - levam ao sofrimento e a estratégias desadaptativas, que podem influenciar comportamentos de autogerenciamento da doença, entre eles a adesão ao tratamento (Reynolds et al., 2009; Seidl \& Faustino, 2014).

Segundo o modelo de autorregulação, a adesão à TARV é diretamente influenciada pelas experiências relacionadas à doença, interações sociais, fontes de informação e processos cognitivo-afetivos (Rosa, 2013). Por exemplo, estudos demonstraram que a percepção de grande número de sintomas associados ao HIV esteve associada a pior adesão ao tratamento antirretroviral, o que pode ser explicado pelo fato do tratamento insatisfatório predispor os indivíduos a sintomas da aids e doenças oportunistas (Cooper, Gellaitry, Hankins, Fisher, \& Horne, 2009; Gonzalez et al., 2007). Ressalta-se que a percepção de doença no que tange ao HIV/aids, utilizando-se o referencial teórico do Modelo de Autorregulação de Leventhal, foi pouco pesquisada no Brasil. Desta forma, entende-se que estudos brasileiros sobre esse tema são de extrema relevância para maior compreensão do papel das cognições e emoções no gerenciamento da doença, podendo servir de embasamento para propostas futuras de intervenção voltadas à população soropositiva. Nessa perspectiva, o objetivo dessa pesquisa foi investigar a associação entre percepção de doença e ansiedade, depressão e autoeficácia em pessoas que vivem com HIV/aids.

\section{Método}

Trata-se de estudo exploratório, com delineamento transversal, correlacional, com o propósito de explorar associações entre as variáveis investigadas.

\section{Participantes}

A amostra foi constituída de 28 pessoas com diagnóstico de HIV/aids em tratamento antirretroviral. Na seleção dos participantes não foi feita distinção entre sexo, raça, situação conjugal, condição socioeconômica e tempo de doença. Os participantes eram maiores de 18 anos e alfabetizados. Foram excluídos do estudo os pacientes que não consentiram em participar, bem como indivíduos com comprometimento intelectual ou 
outra limitação cognitiva que impossibilitasse a adequada compreensão e preenchimento dos instrumentos da pesquisa.

Dezenove participantes eram do sexo masculino $(67,9 \%)$. A média de idade dos participantes foi de 42,4 anos $(D P=8,5 ;$ mínimo= 23; máximo $=56)$. A escolaridade mais frequente foi o ensino médio completo (46,4\%), seguido de ensino fundamental incompleto (14,3\%). Com relação à situação conjugal, $64,3 \%$ afirmaram que não tinham cônjuge ou companheiro(a). Sobre a situação laboral, a maioria não estava trabalhando $(57,1 \%)$, mas prevaleceram rendas superiores a três salários mínimos $(60,7 \%)$. Por fim, $85,7 \%$ disseram que tinham uma religião vinculada a uma doutrina específica.

A metade dos participantes tinha mais de dez anos de diagnóstico. A maioria $(57,2 \%)$ já havia sido internada em decorrência da enfermidade e negou a presença de comorbidades $(64,3 \%)$. Sobre o tratamento antirretroviral, $67,9 \%$ dos participantes começaram a fazer uso dos medicamentos nos últimos dez anos, havendo autorrelato de boa adesão $(82,1 \%)$. Houve menção à existência de atendimento psicológico $(78,6 \%)$ e/ou psiquiátrico $(32,1 \%)$ associado ao diagnóstico e à vivência da soropositividade. $\mathrm{O}$ detalhamento dos dados sociodemográficos e médico-clínicos pode ser visualizados na Tabela 1.

\section{Local}

O estudo foi realizado em um serviço público que oferece assistência médica e psicossocial (psicologia e serviço social) a pessoas vivendo com HIV/aids, em nível ambulatorial e de internação, que funciona em um hospital universitário e integra a rede do Sistema Único de Saúde do Distrito Federal.

\section{Instrumentos}

Questionário Sociodemográfico. Instrumento elaborado para o estudo voltado para a caracterização dos participantes quanto às seguintes informações: sexo, idade, situação conjugal, escolaridade, situaçãolaboral,rendafamiliare religião.

Questionário Médico-Clínico. Instrumento elaborado para o estudo para a coleta de dados sobre tempo de diagnóstico, internações associadas ao HIV/aids, presença de comorbidades, tempo de tratamento antirretroviral, histórico de atendimento psicológico e/ou psiquiátrico relacionado ao HIV/aids e adesão ao tratamento autorrelatada. A adesão ao tratamento foi avaliada por uma pergunta aberta na qual os participantes respondiam se seguiam as recomendações médicas (sim ou não). Essas informações visavam à caracterização médico-clínica dos participantes.

Questionário de Percepção de Doenças Versão Breve (Brief IPQ). Questionário composto por nove itens, adaptado e validado no Brasil por Nogueira (2012), a partir da versão em língua inglesa (Broadbent et al., 2006). Sete itens são avaliados utilizando uma escala de 0-10. São três itens para avaliação da representação cognitiva da doença: controle individual (item 2), conrole do tratamento (item 3) e compreensão (item 6); e quatro itens avaliam a representação emocional: consequências (item 1), identidade (item 4), preocupação (item 5) e emoções (item 7). A avaliação da dimensão temporal (item 8) é feita por uma pergunta aberta na qual é perguntado sobre a percepção da pessoa acerca da duração da enfermidade. Já a avaliação da representação causal (item 9) é feita por uma pergunta aberta na qual os pacientes são interrogados sobre os fatores causais mais importantes associados à doença, segundo a sua percepção. As respostas relacionadas a estes dois últimos itens são analisadas qualitativamente mediante a categorização dos relatos, podendo ser computada a frequência das respostas. Para calcular o escore dos sete itens respondidos mediante escala Likert, inverte-se as respostas dos itens 2, 3 e 6 e adiciona-se os escores dos itens 1, 4, 5 e 7 . A amplitude do escore global do instrumento é de 0 a 70: quanto mais próximo de zero menor a percepção de ameaça da doença e quanto mais próximo de 70 , maior a percepção de ameaça imposta pela enfermidade. Assim, um escore alto reflete uma maior percepção de ameaça da doença. $\mathrm{O}$ alfa de Cronbach da versão brasileira referente aos sete itens é igual a 0,64.

Escala Hospitalar de Ansiedade e Depressão (HADS). Instrumento adaptado e validado 
Tabela 1

Detalhamento dos Dados Sociodemográficos e Médico-Clínicos

\begin{tabular}{|c|c|c|c|}
\hline Variáveis sociodemográficas e médico-clínicas & $\begin{array}{l}\text { Amostra global } \\
\qquad(N=28)\end{array}$ & $\begin{array}{l}\text { Sexo feminino } \\
\quad(n=9)\end{array}$ & $\begin{array}{l}\text { Sexo masculino } \\
(n=19)\end{array}$ \\
\hline Idade (média) & 42,4 & 45,8 & 40,8 \\
\hline \multicolumn{4}{|l|}{ Escolaridade } \\
\hline Ensino Fundamental Incompleto & $14,3 \%$ & $33,3 \%$ & $5,3 \%$ \\
\hline Ensino Fundamental Completo & $3,6 \%$ & $11,1 \%$ & -- \\
\hline Ensino Médio Incompleto & $14,3 \%$ & $22,2 \%$ & $10,5 \%$ \\
\hline Ensino Médio Completo & $46,4 \%$ & $22,2 \%$ & $57,9 \%$ \\
\hline Ensino Superior Incompleto & $10,7 \%$ & -- & $15,8 \%$ \\
\hline Ensino Superior Completo & $10,7 \%$ & $11,1 \%$ & $10,5 \%$ \\
\hline \multicolumn{4}{|l|}{ Situação conjugal } \\
\hline Com companheiro(a) & $35,7 \%$ & $44,4 \%$ & $31,6 \%$ \\
\hline Sem companheiro(a) & $64,3 \%$ & $55,6 \%$ & $68,4 \%$ \\
\hline \multicolumn{4}{|l|}{ Situação laboral } \\
\hline Trabalhando & $42,9 \%$ & $11,1 \%$ & $57,9 \%$ \\
\hline Não estavam trabalhando & $57,1 \%$ & $88,9 \%$ & $42,1 \%$ \\
\hline \multicolumn{4}{|l|}{ Renda familiar } \\
\hline Até 1 salário mínimo & $21,4 \%$ & $33,3 \%$ & $15,8 \%$ \\
\hline De 2 a 3 salários mínimos & $39,3 \%$ & $55,6 \%$ & $31,6 \%$ \\
\hline Mais de 3 salários mínimos & $39,3 \%$ & $11,1 \%$ & $52,7 \%$ \\
\hline \multicolumn{4}{|l|}{ Religião } \\
\hline Com religião específica & $85,7 \%$ & $100 \%$ & $78,9 \%$ \\
\hline Com crenças sem religião & $14,3 \%$ & -- & $21,1 \%$ \\
\hline \multicolumn{4}{|l|}{ Tempo de diagnóstico } \\
\hline Mais de 10 anos & $50 \%$ & $55,6 \%$ & $47,4 \%$ \\
\hline Menos de 10 anos & $50 \%$ & $44,4 \%$ & $52,6 \%$ \\
\hline Com internações devido ao HIV & $57,1 \%$ & $88,9 \%$ & $42,1 \%$ \\
\hline Com comorbidades & $35,7 \%$ & $55,6 \%$ & $26,3 \%$ \\
\hline \multicolumn{4}{|l|}{ Tempo de TARV } \\
\hline Mais de 10 anos & $32,1 \%$ & $22,2 \%$ & $36,8 \%$ \\
\hline Menos de 10 anos & $67,9 \%$ & $77,8 \%$ & $63,2 \%$ \\
\hline Com autorrelato de adesão & $82,1 \%$ & $88,9 \%$ & $78,9 \%$ \\
\hline Teve atendimento psicológico & $78,6 \%$ & $88,9 \%$ & $73,7 \%$ \\
\hline Teve atendimento psiquiátrico & $32,1 \%$ & $22,2 \%$ & $36,8 \%$ \\
\hline
\end{tabular}

para a população brasileira composto por 14 itens, dos quais sete são voltados para a avaliação da ansiedade (HADS-A; alfa de Cronbach $=0,79$ ) e sete para a depressão (HADS-D; alfa de Cronbach $=0,84$ ). Os itens são pontuados de zero a três, sendo que os escores podem variar de zero a 21 pontos para cada subescala. Pessoas que obtêm escore igual ou maior a nove em cada subescala são considerados com ansiedade e/ou com depressão (Marcolino et al., 2007).

Escala de Autoeficácia para Seguir a Prescrição Antirretroviral. Instrumento elaborado e validado por J. C. C. Leite, Drachler, Centeno, Pinheiro e Amato (2002) que avalia a autoefi- 
cácia para seguir o tratamento antirretroviral. Trata-se de uma escala unifatorial, com 22 itens. As respostas são dadas em escala Likert, variando de 0 (com certeza não vou tomar) a 4 (vou tomar com certeza). O instrumento possui boas propriedades psicométricas, com validade de construto e indicador de consistência interna (alfa de Cronbach $=0,96$ ) considerados adequados para medir a autoeficácia para tratamento antirretroviral. Para computar o escore final se divide o valor resultante do somatório dos itens pelo número de itens, sendo que este varia de zero a quatro: quanto maior o escore, ou seja, quanto mais próximo de quatro, maior a autoeficácia percebida para tomar os medicamentos antirretrovirais.

\section{Procedimentos}

Os participantes foram selecionados por conveniência, convidados em sala de espera do ambulatório de infectologia de um hospital universitário. Inicialmente, identificou-se nos prontuários dos pacientes aqueles que tinham diagnóstico de HIV e estavam aguardando a consulta médica. Em seguida foi realizado o convite para participação na pesquisa. Aqueles que aceitaram participar do estudo assinaram o Termo de Consentimento Livre e Esclarecido e responderam de forma individual e autoaplicada aos questionários sociodemográfico e médicoclínico, Brief IPQ, Escala de Autoeficácia para Seguir Prescrição Antirretroviral e HADS.

O estudo foi aprovado pelo Comitê de Ética em Pesquisa da Faculdade de Ciências da Saúde da Universidade de Brasília (parecer $n^{\circ} 331.052$ ).

\section{Análise dos Dados}

Foi conduzida análise dos dados por meio do Statistical Package for the Social Sciences (versão 20), sendo realizadas, inicialmente, análises descritivas das variáveis investigadas. Para investigar a existência de correlações entre percepção de doença e ansiedade, depressão e autoeficácia conduziu-se o teste de correlação de Spearman, teste também usado para avaliar a existência de correlação entre idade e percepção de doença. Para investigar associações entre percepção de doença e sexo, situação conjugal, situação ocupacional, comorbidades e adesão ao tratamento, foi utilizado o teste de Mann-Whitney. Já o teste Kruskal-Wallis foi empregado para avaliar associações entre percepção de doença e escolaridade, renda, tempo de diagnóstico, número de internações e religião. Ressalta-se que também foi realizada análise qualitativa das respostas acerca da dimensão temporal (item 8) e causas da doença (item 9) do Brief IPQ, mediante a classificação das mesmas em categorias.

\section{Resultados}

\section{Percepção de Doença}

Sobre a percepção de doença, 40,3\% dos participantes apresentaram uma percepção de ameaça relevante decorrente da enfermidade. Foi investigada a ocorrência de associação entre percepção de doença e as variáveis sociodemográficas e médico-clínicas: pessoas com relato de boa adesão parecem diferir significativamente daquelas que relataram má adesão com relação à percepção de doença $(U=6,00, p<0,001)$. Não foram evidenciadas associações relevantes entre percepção de doença e as demais variáveis analisadas.

A categorização das respostas referentes à dimensão temporal indicou maior frequência de crenças de que a doença irá durar por toda a vida (39,3\%), seguido de pessoas que relataram não saber quanto tempo a doença iria durar, alegando que a duração dependia da medicina, de Deus ou da descoberta da cura $(28,6 \%)$. Outros referiram que a doença iria durar pouco tempo, justificando que a descoberta da cura ocorreria em breve (14,3\%). Outras respostas somaram 17,8\%: crença de que já está curado por intervenção divina; menção do número de anos de vida restantes de maneira precisa (30 anos com boa adesão; de 12 a 15 anos); afirmação de que não pensa no assunto e uma pessoa não respondeu à pergunta.

Com relação às causas relacionadas à infecção pelo HIV, as categorias identificadas com base nos relatos dos participantes foram: 
relações sexuais sem preservativo $(39,3 \%)$, irresponsabilidade $(17,8 \%)$, falta de informação $(17,8 \%)$ e múltiplos parceiros $(17,8 \%)$.

\section{Ansiedade e Depressão}

Pouco menos da metade dos participantes apresentou escores indicativos de ansiedade $(46,4 \%)$ ou depressão $(46,4 \%)$. Observou-se correlação positiva estatisticamente significativa entre percepção de doença e ansiedade $(r \mathrm{~s}=$ $0,60, p<0,01)$ e depressão $(r \mathrm{~s}=0,65, p<0,01)$. Assim, quanto maior a percepção de ameaça imposta pela enfermidade maior a frequência de sintomas de ansiedade ou depressão.

Com relação aos componentes de percepção de doença - aspectos mensurados pelos sete itens referentes às representações cognitiva e emocional - evidenciou-se correlação estatisticamente significativa entre ansiedade e identidade (item $4 ; r \mathrm{~s}=0,41, p<0,05$ ), preocupações (item $5 ; r \mathrm{~s}=$ $0,49, p<0,01$ ) e emoções (item $7 ; r \mathrm{~s}=0,82, p<$ $0,01)$. Desta forma, quanto mais sintomas graves da doença são percebidos, quanto mais preocupações relacionadas à enfermidade o indivíduo possui e quanto maior o impacto emocional da doença na vida da pessoa, maiores os sintomas de ansiedade relatados, respectivamente.

Também foram encontradas correlações estatisticamente significativas entre os componentes de percepção de doença e depressão no que tange às consequências da doença (item $1 ; r \mathrm{~s}=$
$0,40, p<0,05$ ), identidade (item $4 ; r \mathrm{~s}=0,47$, $p<0,05$ ), preocupações (item $5 ; r \mathrm{~s}=0,50, p<$ 0,01 ) e emoções (item 7; $r \mathrm{~s}=0,71, p<0,05$ ). Assim, um número maior de sintomas de depressão associou-se à percepção de piores consequências decorrentes da doença, à presença de sintomas graves, maiores preocupações e considerável impacto emocional, respectivamente.

\section{Autoeficácia}

Observou-se elevada autoeficácia percebida para tomar os medicamentos antirretrovirais na amostra analisada, sendo encontrado um escore médio de 3,53 $(D P=0,67)$ nesta variável. Foi identificada correlação negativa estatisticamente significativa entre percepção de doença e autoeficácia $(r \mathrm{~s}=-0,41, p<0,05)$, indicando que quanto menor a autoeficácia percebida para tomar os antirretrovirais, maior a percepção de ameaça imposta pela doença. A análise correlacional dos componentes de percepção de doença com essa variável, evidenciou associações entre autoeficácia e controle pessoal (item $2 ; r \mathrm{~s}=0,54$, $p<0,01)$ e controle do tratamento (item $3 ; r \mathrm{~s}=$ $0,50, p<0,01)$. Desta maneira, quanto maior a percepção de autoeficácia para tomar os medicamentos antirretrovirais, maior a sensação de controle sobre a doença e maior é a confiança na eficácia da medicação. O resumo das associações entre as variáveis investigadas e percepção de doença pode ser visualizado na Tabela 2.

Tabela 2

Correlações entre Percepção de Doença (Escore Total e Componentes) e Ansiedade, Depressão e Autoeficácia

\begin{tabular}{lccccccc}
\hline Variáveis & $\begin{array}{c}\text { Percepção } \\
\text { de doença }\end{array}$ & Consequências & $\begin{array}{c}\text { Controle } \\
\text { pessoal }\end{array}$ & $\begin{array}{c}\text { Controle do } \\
\text { tratamento }\end{array}$ & Identidade & Preocupações & Emoções \\
\hline Ansiedade & $0,60^{* *}$ & 0,19 & 0,29 & 0,32 & $0,41^{*}$ & $0,49 * *$ & $0,82^{* *}$ \\
Depressão & $0,65^{* *}$ & $0,40^{*}$ & 0,25 & 0,20 & $0,47 *$ & $0,50^{* *}$ & $0,71^{* *}$ \\
Autoeficácia & $-0,41^{*}$ & $-0,23$ & $0,54 * *$ & $0,50 * *$ & $-0,11$ & $-0,16$ & $-0,12$ \\
\hline
\end{tabular}

Nota. O componente "compreensão" não apresentou correlação estatisticamente significativa com as variáveis investigadas. $* p<0,05 ; * * p<0,01$.

\section{Discussão}

A maioria dos participantes do estudo não apresentou uma percepção de ameaça relevante imposta pela enfermidade. Acredita-se que isso possa decorrer da eficácia da TARV, bem como ao acesso universal e gratuito aos medicamentos no Brasil. Assim, as PVHA atualmente tem 
acesso à informação de que é possível viver bem com a doença, de manter a qualidade de vida, desde que haja adesão satisfatória ao tratamento antirretroviral (Seidl \& Faustino, 2014). Ademais, menor percepção de ameaça da enfermidade esteve correlacionada com maior autoeficácia percebida para seguir o tratamento, dado que é coerente com a frequência elevada de autorrelatos de adesão satisfatória ao tratamento $(82,1 \%)$, o que pode contribuir para a construção de uma visão menos ameaçadora da doença. Contudo, cabe lembrar que mesmo diante de boa adesão ao tratamento e da percepção de que a doença não apresenta um ameaça relevante, viver com uma enfermidade crônica como o HIV/aids, ainda estigmatizada, pode acarretar sofrimento psíquico.

Nesse estudo, observou-se que a maioria das pessoas tinha consciência de que a doença iria durar pelo resto da vida, tal como observado em um estudo conduzido na Carolina do Norte. Nessa investigação a maioria dos participantes afirmou que o HIV/aids é uma doença crônica (Kemppainen et al., 2008), sendo que houve também a menção de esperança na cura alimentada por crenças religiosas ou pelos progressos em pesquisas na área. Uma pesquisa polonesa também revelou predomínio de crenças associadas à percepção de cronicidade da aids, o que foi interpretado positivamente pelos autores, acreditando que tal crença poderia favorecer a colaboração com seus médicos e o cumprimento do regime de tratamento (Kossakowska \& Zielazny, 2013).

Sobre a percepção de causalidade da enfermidade, constatou-se que pessoas com HIV/ aids fizeram atribuições predominantemente coerentes com aquelas descritas na literatura: práticas associadas a comportamentos sexuais de risco. Ressalta-se que a principal via de transmissão, desde o início da epidemia, é por relação sexual desprotegida (World Health Organization, 2011). Cabe destacar que são frequentes os relatos de autoculpabilização, raiva direcionada a si mesmo e/ou ao(a) parceiro(a) sexual e sentimentos de arrependimento pela exposição ao vírus devido ao sexo sem preservativo, o que também pode levar a prejuízos no que tange à saúde mental (Ministério da Saúde, 2012; Seidl \& Faustino, 2014).

Foram evidenciadas associações entre percepção de doença e depressão e ansiedade. Segundo Sale (2014), pessoas que apresentam uma percepção de doença negativa são mais propensas a desenvolver depressão. De maneira semelhante ao presente estudo, Slot et al. (2015) identificaram alguns fatores associados ao risco de depressão em pessoas infectadas pelo HIV, entre eles: percepção de que o HIV afeta todos os aspectos da vida (ou seja, percepção de consequências impostas pela doença) e problemas emocionais associados ao diagnóstico de soropositividade, como estresse. Assim, as correlações entre depressão e percepção de doença observadas nesse estudo parecem coerentes, tendo em vista que a percepção de piores consequências associadas ao HIV/aids, preocupações exacerbadas com a enfermidade e efeitos relevantes no estado emocional estiveram associados a maior número de sintomas depressivos.

Cabe ressaltar, que a depressão é uma das alterações psiquiátricas mais comuns em pessoas infectadas pelo HIV (Christo \& Paula, 2008; Ministério da Saúde, 2012; Seidl \& Faustino, 2014; Slot et al., 2015). Contudo, há grande variação na prevalência desse transtorno psicológico em pessoas que vivem com HIV/ aids, com resultados que variam de 0 a $45 \%$, sendo os dados desse estudo superiores àqueles apontados pela literatura $(46,4 \%)$. Todavia, um estudo brasileiro encontrou uma prevalência ainda maior, com 58\% da amostra com depressão moderada a severa (Homero et al., 2008). Tal variabilidade nos níveis de prevalência pode ser justificada por fatores como: população estudada, instrumentos de avaliação utilizados, local de realização da pesquisa e estágio da doença (Christo \& Paula, 2008).

O transtorno depressivo pode ser desencadeado devido à inexistência de cura para o HIV/ aids, pelo sentimento de falta de controle sobre o futuro, pelos limites impostos pela doença, vivência ou medo de preconceito, autoculpabilização e recriminação por haver-se exposto ao vírus, sentimentos de culpa pela possibilidade de ter infectado alguém involuntariamente, além 
do preconceito e discriminação vivenciados em diferentes esferas da vida (Ministério da Saúde, 2012; Seidl \& Faustino, 2014). Em função disso, muitas pessoas soropositivas adotam condutas de isolamento devido ao medo de rejeição, enfrentando dificuldades em iniciar novos relacionamentos afetivos, sociais e sexuais, bem como de revelar o diagnóstico. Em consequência, muitos sofrem redução da autoestima, sentimentos de falta de pertencimento e perda da identidade psicológica e social, o que pode desencadear sofrimento psíquico e transtorno mental, como a depressão (Ferris et al., 2015; Herrmann et al., 2013; Ministério da Saúde, 2012). Assim, acredita-se que o diagnóstico e o tratamento de sintomas depressivos em pessoas infectadas pelo HIV pode levar a uma maior adesão ao tratamento, melhora da qualidade de vida e adaptação psicossocial (Christo \& Paula, 2008; Ministério da Saúde, 2012).

A ansiedade também é comum em pessoas com HIV, sendo frequente entre os participantes do estudo (46,4\%). É importante salientar que aquelas que já possuíam sintomas desse transtorno anteriormente à infecção podem ter os mesmos potencializados devido a fatores estressantes relacionados ao diagnóstico e à vivência da soropositividade, incluindo: preocupações com a progressão da doença, medo de infectar outras pessoas e receio de rejeição em diversos aspectos da sua vida, incluindo o contexto social, afetivo/sexual, familiar e ocupacional (Campos \& Thomason, 2007; Christo \& Paula, 2008). Isso também é coerente com os resultados observados entre maior percepção de ameaça da doença e ansiedade, pois a percepção de mais sintomas pode ser indicativa de progressão da doença, o que pode potencializar as preocupações já existentes associadas às dificuldades vivenciadas pelas PVHA e acarretar um impacto negativo no estado emocional.

Evidências da associação entre ansiedade e depressão e componentes da percepção de doença com base no modelo de Leventhal foram verificadas em outras enfermidades. Em estudo conduzido com pessoas que tinham tuberculose foi observada associação entre ansiedade e depressão e a percepção de consequências mais sérias e de menor controle da doença (Husain, Dearman, Chaudhry, Rizvi, \& Waheed, 2008). Tais achados são parcialmente condizentes com o presente estudo, já que correlações entre depressão e percepção de consequências mais sérias da doença foram observadas. Todavia, são enfermidades com características distintas, apesar de ambas serem infecciosas, pois a tuberculose é uma doença aguda e curável. Assim, cada enfermidade impacta de modo particular o funcionamento biopsicossocial das pessoas, o que limita generalizações para outros contextos de adoecimento.

Outra variável investigada no estudo foi a autoeficácia para tomar os antirretrovirais, sendo de considerável importância no contexto de assistência às PVHA. Pesquisas indicam que pessoas com elevada autoeficácia são mais propensas a apresentar uma adesão ao tratamento satisfatória (Bofill et al., 2014; Houston et al., 2015; Seidl et al., 2007). No presente estudo esta variável correlacionou-se com percepção de doença, especialmente com percepção de controle pessoal (crença do indivíduo na capacidade de controlar a doença) e controle do tratamento (crença na eficácia do tratamento), associação também descrita em um estudo multicêntrico. Nessa investigação, realizada em Taiwan, Noruega, Porto Rico, Colômbia e Estados Unidos, a percepção de que pouco poderia ser feito para controlar a doença esteve associado com menos ações de autocuidado efetivas (Reynolds et al., 2009). Associação entre autoeficácia e percepção de controle da doença também foi identificada em outras enfermidades, como doença cardíaca (Lau-Walker, 2006; Paryad, Hosseinzade, Kazemnejad, \& Asiri, 2013) e diabetes em adolescentes (Griva, Myers, \& Newman, 2000). Isso é bastante coerente, já que pessoas que se sentem capazes de prosseguir e acreditam que o tratamento ajuda no controle da doença são naturalmente mais propensas a ingerir a medicação conforme recomendação médica. Contudo, cabe lembrar, que é necessária parcimônia na generalização de tais resultados por se tratarem de enfermidades distintas e de estudos estrangeiros que podem não refletir a realidade brasileira. Isso porque questões culturais, bem como o acesso a 
tratamentos e serviços de saúde podem variar em diferentes países, e afetar a percepção de doença, especialmente a de controle da mesma.

Acredita-se na importância de intervenções psicológicas voltadas a mudanças na percepção de doença, já que a construção de um novo repertório cognitivo e comportamental, incluindo a implementação de estratégias de enfrentamento mais adaptativas, contribua para a redução de sintomas de depressão, ansiedade e para o aumento da autoeficácia para tomar os antirretrovirais. Uma intervenção realizada em PVHA em Portugal parece indicar que este pode ser um caminho viável (Rosa, 2013): foi desenvolvida uma intervenção em grupo baseada no Modelo de Autorregulação de Leventhal que acarretou em melhora da autoeficácia para tomar os medicamentos antirretrovirais. Estudos para investigar se os efeitos benéficos dessa modalidade de intervenção se mantêm ao longo do tempo e se tais benefícios seriam observados caso esta intervenção fosse replicada em outras realidades, como no Brasil, merecem ser desenvolvidos.

Como limitações da pesquisa citam-se: número reduzido de participantes e seleção por conveniência, bem como coleta de dados em um único serviço que presta assistência a PVHA, o que impossibilita generalizações acerca dos resultados do presente estudo. Assim, sugere-se que pesquisas futuras investiguem a associação entre tais variáveis em uma amostra maior, em outras regiões do país, bem como em outras enfermidades crônicas. Além disso, acredita-se na importância da investigação da associação entre percepção de doença e outras variáveis não abordadas nessa pesquisa, como suporte social, estratégias de enfrentamento e qualidade de vida.

\section{Considerações Finais}

A percepção de doença é uma variável importante a ser considerada na prática e na pesquisa em doenças crônicas, incluindo o HIV/aids. Os resultados encontrados nesse estudo reforçam a sua relevância, visto que foi evidenciada associação entre a mesma e ansiedade, depressão e autoeficácia para tomar os antirretrovirais em PVHA.
Assim, o presente estudo tem o mérito de contribuir na divulgação do construto percepção de doença, baseado no Modelo de Autorregulação de Leventhal, favorecendo sua compreensão e eventual utilização por profissionais e pesquisadores brasileiros. Espera-se que esse estudo contribua também para a ampliação de conhecimentos sobre a associação entre percepção de doença e variáveis psicológicas, servindo de estímulo para que sejam desenvolvidas intervenções psicológicas voltadas para a promoção de mudanças na percepção de doença.

\section{Referências}

Alencar, T. M. D., Nemes, M. I. B., \& Velloso, M. A. (2008). Transformações da "aids aguda" para a "aids crônica": Percepção corporal e intervenções cirúrgicas entre pessoas vivendo com HIV e aids. Ciência \& Saúde Coletiva, 13(6), 18411849. doi:10.1590/S1413-81232008000600019

Arran, N., Craufurd, D., \& Simpson, J. (2014). Illness perceptions, coping styles and psychological distress in adults with Huntington's disease. Psychology, Health \& Medicine, 19(2), 169179. doi:10.1080/13548506.2013.802355

Bofill, L. M., Lopez, M., Dorigo, A., Bordato, A., Lucas, M., Cabanillas, G. F., ... Jones, D. (2014). Patient-provider perceptions on engagement in HIV care in Argentina. AIDS Care, 26(5), 602-607. doi:10.1080/09540121.2013.844767

Broadbent, E., Petrie, K. J., Main, J., \& Weinman, J. (2006). The Brief Illness Perception Questionnaire. Journal of Psychosomatic Research, 60, 631-637. doi:10.1016/j.jpsychores.2005.10.020

Brooke, J. (2013). Impact of variables within the Common Sense Model of Self-Regulation on health outcomes: A systematic review and meta-analysis. Paper presented at the Twenty-four International Nursing Research Conference, Prague, Czech Republic. Retrieved from https://www2. rcn.org.uk/_data/assets/pdf_file/0004/511438/ Research2013We03.pdf

Calvetti, P. U., Muller, M. C., \& Nunes, M. L. T. (2007). Psicologia da saúde e psicologia positiva: Perspectivas e desafios. Psicologia Ciência e Profissão, 27(4), 706-717. doi:10.1590/S141498932007000400011

Cameron, L., \& Leventhal, H. (2003). Self-regulation, health, and illness: A overview. In L. D. 
Cameron \& H. Leventhal (Eds.), The self-regulation of health and illness behavior (pp. 1-14). New York: Routledge.

Campos, P. E., \& Thomason, B. (2007). Intervenções cognitivo-comportamentais com pessoas com HIV/aids. In V. E. Caballo (Ed.), Manual para o tratamento cognitivo-comportamental dos transtornos psicológicos da atualidade (pp. 441-461). São Paulo, SP: Santos.

Chen, S. L., Tsai, J. C., \& Lee, W. L. (2009). The impact of illness perception on adherence to therapeutic regimens of patients with hypertension in Taiwan. Journal of Clinical Nursing, 18(15), 2234-2244. doi:10.1111/j.13652702.2008.02706.x

Christo, P. P., \& Paula, G. (2008). Aspectos neuropsiquiátricos e neuropsicológicos da infecção pelo HIV e da aids. In D. Fuentes, L. F. Malloy-Diniz, C. H. P. Camargo, \& R. M. Cosenza (Eds.), Neuropsicologia: Teoria e prática (pp. $335-$ 355). Porto Alegre, RS: Artmed.

Colleto, M., \& Câmara, S. (2009). Estratégias de coping e percepção da doença em pais de crianças com doença crônica: O contexto do cuidador. Diversitas: Perspectivas em Psicología, 5(1), 97-110.

Cooper, V., Gellaitry, G., Hankins, M., Fisher, M., \& Horne, R. (2009). The influence of symptom experiences and attributions on adherence to highly active anti-retroviral therapy (HAART): A six-month prospective, follow-up study. AIDS Care, 21(4), 520-528. doi:10.1080/09540120802301824

Ferris, F. N., McDonald, S., Conroy, R. R., Byrne, E., Mallouris, C., Hodgson, I., \& Larkan, F. (2015). "An unspoken world of unspoken things": A study identifying and exploring core beliefs auderlying self-stigma among people living with HIV and aids in Ireland. Swiss Medical Weekly. Retrieved from http://www.ncbi.nlm.nih.gov/ pubmed/25768695

Figueiras, M. J. (2008). A relevância dos modelos de senso-comum da doença para a adesão terapêutica. Revista Factores de Risco, 11, 38-41.

Galvão, M. T. G., Gir, E., Fiuza, M. L. T., Cunha, G. H., \& Reis, R. K. (2013). Niveis de ansiedade e depressão e adesão aos antirretrovirais de pessoas com HIV/AIDS. Trabalho apresentado no Décimo Sétimo Seminário Nacional de Pesquisa em Enfermagem, Natal, RN, Brasil. Resumo recuperado em http://www.abeneventos.com.br/ anais_senpe/17senpe/pdf/0424co.pdf

Gonzalez, J. S., Penedo, F. J., Llabre, M. M., Duran, R. E., Antoni, M. H., Schneiderman, N., \& Horne, R. (2007). Physical symptoms, beliefs about medications, negative mood, and long-term HIV medication adherence. Annals of Behavioral Medicine, 34(1), 46-55. doi:10.1080/08836610701495565

Griva, K., Myers, L. B., \& Newman, S. (2000). Illness perceptions and self efficacy beliefs in adolescents and young adults with insulin dependent diabetes mellitus. Psychology \& Health, 15(6), 733-750. doi:10.1080/08870440008405578

Herrmann, S., McKinnon, E., Hyland, N. B., Lalanne, C., Mallal, S., Nolan, D., ...Duracinsky, M. (2013). HIV-related stigma and physical symptoms have a persistent influence on healthrelated quality of life in Australians with HIV infection. Health and Quality of Life Outcomes, 11(56), 1-13. doi:10.1186/1477-7525-11-56

Homero, W. M., Santos, W. S., Roxo, M., Atti, V., Paim, P., Moschen, T., ...Michelin, L. (2008). Prevalência de ansiedade e depressão em pacientes com HIVIAIDS. Trabalho apresentado no Vigésimo Salão de Iniciação Científica da Universidade Federal do Rio Grande do Sul, Porto Alegre, RS, Brasil. Resumo recuperado em http://www.lume.ufrgs.br/handle/10183/50919

Houston, E., Mikrut, C., Guy, A., Fominaya, A. W., Tatum, A. K., Kim, J. H., \& Brown, A. (2015). Another look at depressive symptoms and antiretroviral therapy adherence: The role of treatment self-efficacy. Journal of Health Psychol$o g y$. Retrieved from http://www.ncbi.nlm.nih. gov/pubmed/25712489

Husain, M. O., Dearman, S. P., Chaudhry, I. B., Rizvi, N., \& Waheed, W. (2008). The relationship between anxiety, depression and illness perception in tuberculosis patients in Pakistan. Clinical Practice and Epidemiology in Mental Health, 4(4), 1-5. doi:10.1186/1745-0179-4-4

Joint United Nations Programme on HIV/AIDS. (2014). Fast-track end the aids epidemic by 2030. Retrieved from http://www.unaids.org.br/ biblioteca/JC2686 WAD2014report en.pdf

Keeling, M., Bambrough, J., \& Simpson, J. (2013). Depression, anxiety and positive affect in people diagnosed with low-grade tumours: The role of illness perception. Psychooncology, 22(6), 1421-1427. doi:10.1002/pon.3158 
Kemppainen, J., Kim-Godwin, R., Reynolds, N. R., \& Spencer, V. S. (2008). Beliefs about HIV disease and medications adherence in persons living with HIV/aids in rural southeastern North Carolina. The Journal of the Association of Nurses in Aids Care, 19(2), 127-136. doi:10.1016/j.jana.2007.08.006

Kossakowska, M., \& Zielazny, P. (2013). Illness perceptions and benefit finding among people with HIV/ AIDS. Postepy Psychiatrii i Psychologii, 22, 177-186.

Lau-Walker, M. (2006). Predicting self-efficacy using illness perception components: A patient survey. British Journal of Health Psychology, 11(4), 643-661. doi:10.1348/135910705X72802

Leite, C. M. (2011). A self-regulation model of depression: Content of cognitive representations and prediction of treatment seeking (Doctoral dissertation). Retrieved from http://ir.lib.uwo.ca/ cgi/viewcontent.cgi? article $=1487 \&$ context $=$ etd

Leite, J. C. C., Drachler, M. L., Centeno, M. O., Pinheiro, C. A. T., \& Amato, V. L. (2002). Desenvolvimento de uma escala de auto-eficácia para adesão ao tratamento antirretroviral. Psicologia: Reflexão e Crítica, 15, 121-133.

Marcolino, J. A. M., Mathias, L. A. S. T., Piccinini, L., Filho, Guaratini, A. A., Suzuki, F. M., \& Alli, L. A. C. (2007). Escala hospitalar de ansiedade e depressão: Estudo da validade de critério e da confiabilidade com pacientes no pré-operatório. Revista Brasileira de Anestesiologia, 57(1), 5262. doi:10.1590/S0034-70942007000100006

Ministério da Saúde. (2008). Manual de adesão ao tratamento para pessoas vivendo com HIV e aids. Brasília, DF: Secretaria de Vigilância à Saúde.

Ministério da Saúde. (2012). Atenção em saúde mental nos serviços especializados em DST/Aids. Brasília, DF: Secretaria de Vigilância à Saúde.

Ministério da Saúde. (2014). Boletim epidemiológico $H I V$-Aids. Brasília, DF: Secretaria de Vigilância à Saúde.

Nogueira, G. S. (2012). Adaptação e validação do Brief Illness Perception Questionnaire (Brief IPQ) para a cultura brasileira (Dissertação de mestrado). Recuperado em http://repositorio. unb.br/bitstream/10482/10777/1/2012 GrazielaSousaNogueira.pdf

Pacheco-Huergo, V., Vildrich, C., Pujol-Ribera, E., Cabezas-Peña, C., Núñez, M., Roura-Olmeda, P., ...Val, J. L. (2012). Percepción em enferme- dades crônicas: validación linguística del Illness Perception Questionnaire Revised y del Brief Illness Perception Questionnaire para la población española. Atención Primaria, 44(5), 280287. doi:10.1016/j.aprim.2010.11.022

Paryad, E., Hosseinzade, T., Kazemnejad, E., \& Asiri, S. (2013). A study of self-efficacy in patients with coronary artery disease and its predictors. Qom University of Medical Sciences Journal, $7(2), 1-6$.

Reynolds, N. R., Eller, L. S., Nicholas, P. K., Corless, I. B., Kirksey, K., Hamilton, M. J., ...Holzemer, W. L. (2009). HIV illness representation as a predictor of self-care management and health outcomes. Aids and Behavior, 13(2), 258-267. doi:10.1007/s10461-007-9297-5

Rosa, A. L. C. J. (2013). Adesão à terapêutica no VIH/SIDA: Efeitos de uma intervenção baseada no modelo de auto-regulação (Dissertação de mestrado). Recuperado em http://repositorio. ispa.pt/handle/10400.12/2742

Sale, S. (2014). Psycho-social and medical variables of depression in HIV/AIDS patients in Kano, Nigeria. Journal of Therapy and Management in HIV Infection, 2, 36-44. doi:10.12970/23090529.2014.02.02.1

Seidl, E. M. F., \& Faustino, Q. M. (2014). Pessoas Vivendo com HIV/aids: Possibilidades de atuação da psicologia. In E. M. F. Seidl \& M. C. O. S Miyazaki (Eds.), Psicologia da saúde: Pesquisa $e$ atuação profissional no contexto de enfermidades crônicas (pp. 21-54). Curitiba, PR: Juruá.

Seidl, E. M. F., Melchíades, A., Farias, V., \& Brito, A. (2007). Pessoas vivendo com HIV/aids: Variáveis associadas à adesão ao tratamento antirretroviral. Cadernos de Saúde Pública, 23(10), 2305-2316. doi:10.1590/S0102311X2007001000006

Sharma, S., Khadga, P., Dhungana, G. P., \& Chitrakar, U. (2013). Medication adherence to antiretroviral therapy among patients visiting antiretroviral therapy center at Tribhuvan University Teaching Hospital, Kathmandu Nepal. Kathmandu University Medical Journal, 11(41), 50-53. doi:10.3126/kumj.v11i1.11027

Silva, S. F. R., Pereira, M. R. P., Motta, R., Neto, Ponte, M. F., Ribeiro, I. F., Costa, P. F. T. F., \& Silva, S. L. (2010). Aids no Brasil: Uma epidemia em transformação. Revista Brasileira de Análises Clínicas, 42(3), 209-212. 
Singh, R. (2011). Psychological model of illness. Newcastle upon Tyne, UK: Cambridge Scholars.

Slot, M., Sodemann, M., Gabel, C., Holmskov, J., Laursen, T., \& Rodkjaer, L. (2015). Factors associated with risk of depression and relevant predictors of screening for depression in clinical practice: A cross-sectional study among HIVinfected individuals in Denmark. HIV Medicine. doi:10.1111/hiv.12223

Sousa, M. R. G., Landeiro, M. J. L., Pires, R., \& Santos, C. (2011). Coping e adesão ao regime terapêutico. Revista de Enfermagem, 4(3), 151-160. doi:10.12707/riii1010

Straub, R. O. (2014). Psicologia da Saúde: Uma abordagem psicossocial (3. ed.). Porto Alegre, RS: Artmed.
Tucker, J. S., Burnam, A., Sherbourne, C. D., Kung, F. Y., \& Gifford, A. L. (2003). Substance use and mental health correlates of nonadherence to antiretroviral medications in a sample of patients with human immunodeficiency virus infection. The American Journal of Medicine, 114, 573580. doi:10.1016/S0002-9343(03)00093-7

World Health Organization. (2011). Prevention and treatment of HIV and other sexually transmitted infections among men who have sex with men and transgender people. Geneva, Switzerland: HIV/Aids Programme.

Recebido: $22 / 01 / 2015$

$1^{a}$ revisão: 27/04/2015

Aceite final: 1\%06/2015 